\title{
The treatment of articular cartilage injuries with mesenchymal stem cells in different animal species
}

\author{
Ilnur Ganiev ${ }^{1 *}$, Natalia Alexandrova ${ }^{1}$, Alexander Aimaletdinov ${ }^{1}$, Catrin Rutland ${ }^{2}$, Albina Malanyeva ${ }^{1}$, \\ Albert Rizvanov $^{1}$ and Elena Zakirova ${ }^{1}$ \\ ${ }^{1}$ Institute of Fundamental Medicine and Biology of Kazan (Volga region) Federal University, Kazan, Russia \\ ${ }^{2}$ School of Veterinary Medicine and Science, College Road, Sutton Bonington, University of Nottingham, Nottingham, UK
}

\begin{abstract}
One of the major problems observed in veterinary practice is articular cartilage injuries in animals. In terms of agriculture, it leads to their culling from the herd, even if they are highly productive animals. With companion animals, owners usually have to decide between euthanasia or long-term sometimes lifelong treatment of the injury by a veterinarian. The use of mesenchymal stem cells (MSCs) for the treatment of cartilage injury in veterinary medicine is based on the good results observed in preclinical studies, where large animals have been used as experimental models to study the regenerative activity of MSCs. According to the literature, MSCs in veterinary medicine have been used to treat cartilage injury of dogs and horses, whereas sheep and goats are generally models for reproducing the disease in preclinical experimental studies.
\end{abstract}

Keywords: Mesenchymal stem cells, Cartilage tissue, Animals, Regeneration, Therapy.

\section{Introduction}

Mesenchymal stem cells (MSCs) are precursors of human and animal tissues. MSCs are detected within, and can be separated from, various sources including bone marrow (BM), adipose tissue (AT), cord tissue (CT), cord blood (CB), Wharton's jelly (WJ), the pulp of milk teeth, the endometrium, and other sources. Modern researches have shown that MSCs process regenerative activities within damaged body tissues (Zakirova et al., 2017, 2019) and can be differentiated into four lines. This therapeutic effect is due to their ability to affect injured tissues with biologically active substances secreted by MSCs (Ragni et al., 2020).

The research into veterinary regenerative medicine has focused mainly on domestic and sporting animals, but a critical reading of the published results, combined with selected articles published for domestic animals, allows us to gain valuable insights about the future use of regenerative medicine in farming. Among all domesticated animals, cattle play a crucial role in the farming economy. There are several diseases, such as mastitis, lameness, and monomeric fractures to limb bones, which can negatively affect the production of meat and milk, in addition to the reduction in reproductive efficiency in cattle. For cows with high economic or genetic potential, these losses create significant costs for the owner, and the owner is obliged to use expensive and effective treatment methods to restore the animal's health (Jean and Anderson, 2014). According to literature data, osteoarthritis (OA) affects about $20 \%$ of dogs (Pigareva, 2016), and in horses, it is one of the most common causes of lameness. It is shown that approximately $33 \%$ of horses have cartilage injury associated with OA (Nifontov, 2009). Therefore, it is important to develop therapeutic approaches to stimulate the regeneration of hyaline tissue for better joint rehabilitation (Juneau et al., 2016).

The aim of this review is to scrutinize the modern achievements of regenerative veterinary medicine in the treatment of joint injuries in animals.

The peculiarities of the structure and regeneration of cartilage tissue concerning MSC use

Articular cartilage is mainly composed of hyaline tissue which in itself is a specialized tissue present in most joints, providing low abrasion and amortization. It also provides a structural and biological barrier between the two bone surfaces, for an unimpeded, even range of motion in the joints. It should be noted that the cartilage is devoid of blood vessels, lymph nodes, and nerves and is generally split into the superficial, middle, and deep zones with calcified cartilage situated next to the subchondral bone (Duarte Campos, 2012) (Fig. 1).

Cartilage tissue mainly comprises $70 \%-80 \%$ of water, $10 \%-15 \%$ of organic substances, and $4 \%-7 \%$ mineral salts. Collagen is found between $50 \%$ and $70 \%$ of the dry matter, which gives tensile strength, and also in proteoglycans, which provide functional resistance to compression (Francis et al., 2018). The thickness of articular cartilage depends on the type of joint and the age of the animal, in addition to the species type. The average thickness of knee cartilage in adult rabbits, sheep, dogs, goats, horses, and humans is $0.3,0.4-0.5$, $0.6-1.3,0.7-1.5,1.5-2.0$, and $2.2-2.5 \mathrm{~mm}$, respectively (Frisbie et al., 2006). Due to its basic structure, 


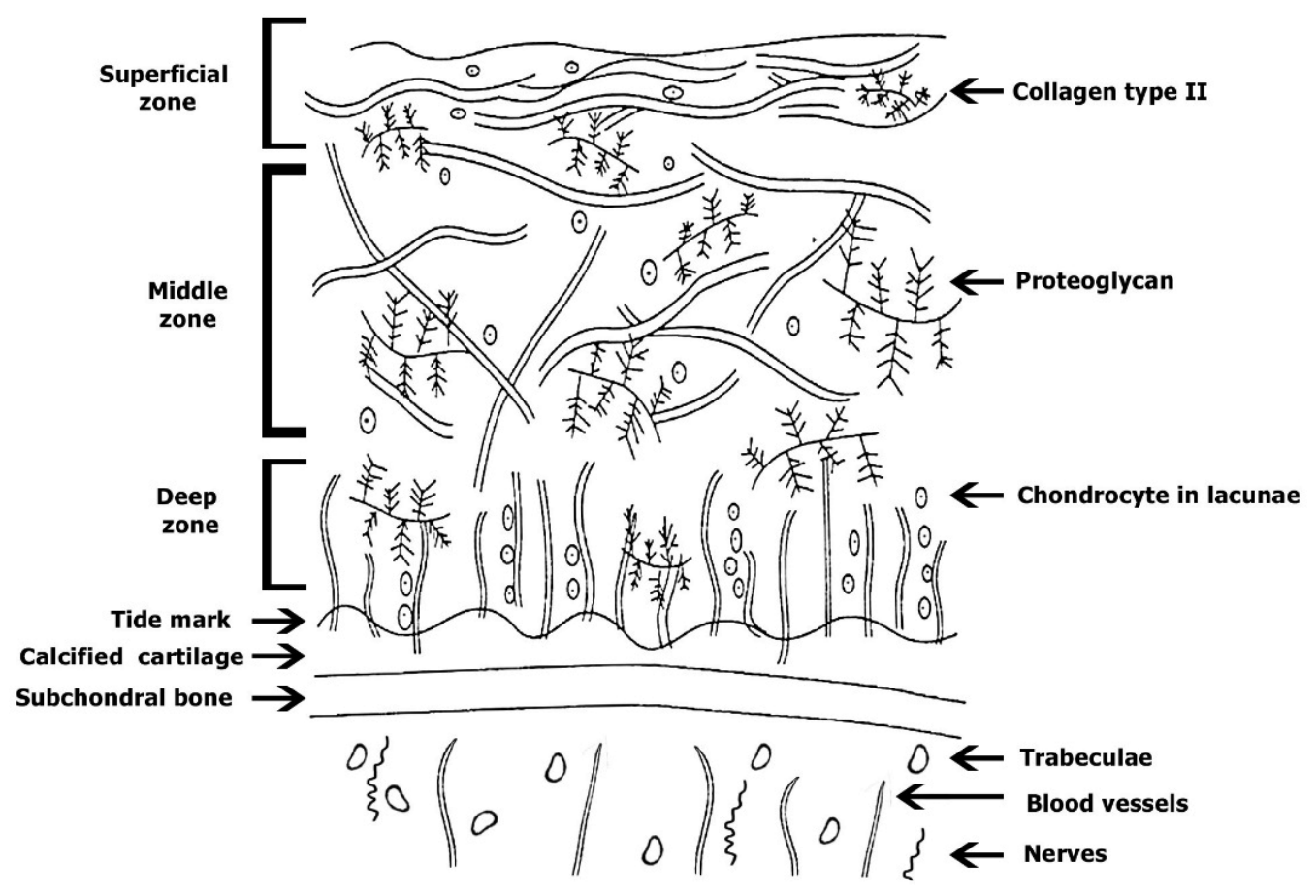

Fig. 1. Structure of cartilage tissue.

damaged hyaline cartilage has low regenerative abilities. This is a serious problem in orthopedics, both with and without treatment; cartilage injuries are filled with fibrous tissue, but this does not have the necessary specialized properties of hyaline cartilage and, therefore, compromises structure and function (Redman et al., 2005). Cartilage defects can penetrate throughout the entire thickness of the cartilage into the subchondral bone, or be limited to the cartilage itself (Gugjoo et al., 2016). Defects penetrating partially through the cartilage do not heal spontaneously due to the absence of a fibrin clump and, consequently, of reparative stem cells, although full thickness injuries heal spontaneously as they are filled in mechanically with structurally diminished fibrous tissues that do not integrate with native cartilage (Tiwary et al., 2014). However, one risk is that if the treatment is ineffective, the injury can lead to OA (Fig. 2). It should be noted that cartilage degeneration and inflammation are key signs of OA, which can affect the entire joint and cause pain, deformation, and loss of function (Toh et al., 2016). Studies on animals show that MSCs obtained from adult tissues are prospective candidates for the treatment of injuries because they have low immunogenicity and high anti-inflammatory potential (Gao et al., 2015; Mount et al., 2015). In vivo studies using MSCs for cartilage therapy have been carried out on almost all domesticated species of mammals, including sheep, goats, dogs, horses, cattle, cats, and pigs. Both allogeneic and autogenic cells have been used in these studies. Researchers have noted that allogeneic MSCs transplanted once or several times are safe and do not cause any reactions from the immune system (Ardanaz et al., 2016; Vega et al., 2015). The cells are detected in vivo in the implantation zone even after 14 weeks (Feng et al., 2018). At present, it is recommended that MSCs be administered after anti-inflammatory therapy during the treatment of injured joint surfaces (Ando et al., 2012; Zayed et al., 2016).

Transplantation of MSCs in sheep and goats for cartilage injury treatment

At present, there are many articles devoted to the treatment of cartilage injury with MSCs originating from BM and AT, as they were extensively used to show the impact of MSCs transplantation in cartilage injury in sheep and goats which were used as models. The methods of administration of MSCs-AT and MSCs-BM are different but both intra-articular and systemic administration is recommended. However, all published studies have shown positive effects and an absence of complications in the animals following the introduction of MSCs (Caminal et al., 2014).

Some researchers recommend that to strengthen the regenerative potential of MSCs-BM, they should be pre-differentiated in the chondrogenic direction. In a model of surgically induced OA in sheep, predifferentiated MSCs-BM were introduced intraarticularly and compared against a positive control group where intact MSCs-BM were introduced and negative control group animals were given a basal environment without cells. According to histological studies in the experimental group, the thickness and 


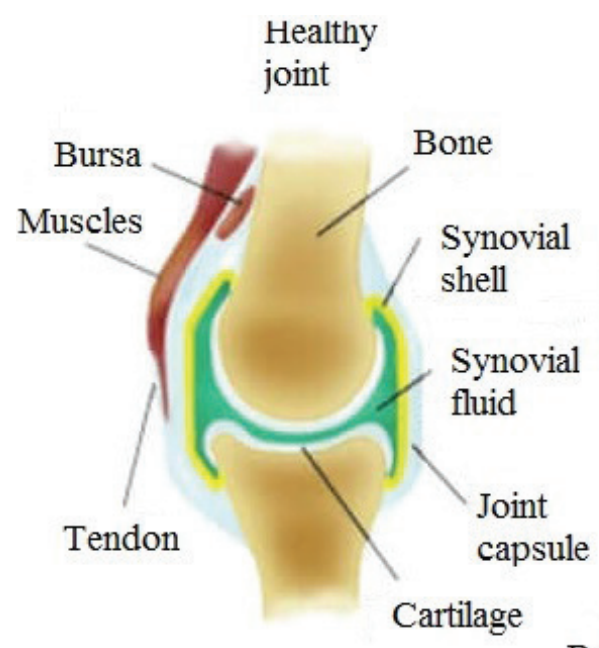

Bones rub against each other
Arthrosis Arthritis

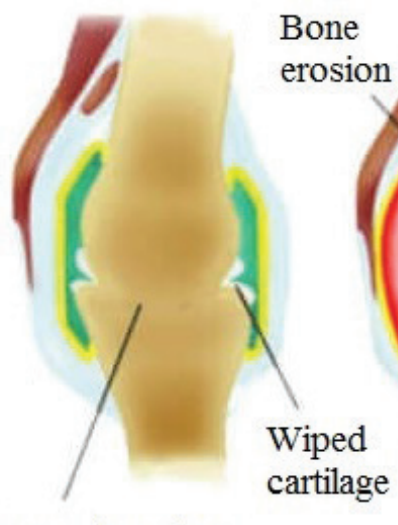

Wiped

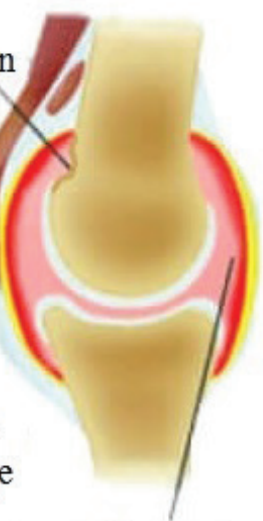

Inflammatory exudate in the joint cavity

\section{Illness progression}

Fig. 2. Development of arthritis.

quality of cartilage tissue were comparable to intact knee joint cartilage. In the positive control group, the researchers also noted positive effects from MSCs$\mathrm{BM}$ introduction, and in the negative control group, severe $\mathrm{OA}$ and meniscus damages were observed. Thus, a single intra-articular injection of intact or pre-differentiated in vitro MSCs-BM may slowdown the progression of OA in the sheep model, but predifferentiated cells showed better results, especially in the process of meniscus regeneration (Al Faqeh et al., 2012).

Bornes et al. (2018) studied the effects of oxygen concentration on the regenerative potential of MSCs$\mathrm{BM}$ differentiated in the chondrogen direction. At the same time, native MSCs-BM was grown on scaffolds (cell matrices) made of hyaluronic acid. Before transplantation, MSCs were injected into the damaged joint and then placed in a differentiation medium and cultured at $21 \%$ oxygen (normoxia) or $3 \%$ (hypoxia) for 4 days. Cells were not transplanted into animals in the control group having damaged joints. According to the results obtained from the experiments, the researchers concluded that when cells were cultured in vitro in a chondrogenic environment in hypoxic samples, the expression of aggrecan and type 2 of collagen increased. However, after in vivo implantation, the difference between normoxic and hypoxic samples on histological morphology was not detected, but in comparison with acellular controls in the two experimental groups, there were higher percentages of filling within injuries with a hyaline-like tissue (Bornes et al., 2018).
Abdalmula et al. (2017) studied the regenerative potential of MSCs which were obtained from the ovary of a sheep on a model of sheep monoarthritis. They received collagen-induced hock monoarthritis of the joint, and then animals were given intravenous MSCs derived from ovarian mesenchyma. As a result of the experiments, a positive regenerative effect of the selected MSCs was shown. The publication notes significant reductions in lameness, pain in the affected joints, and edema as compared to the animals in the control group. Besides, histological studies showed decreased cartilage erosion in the experimental group along with activation of the synovial stromal cells and angiogenesis. These effects were accompanied by a decrease in the infiltration of synovial tissues by CD4 + lymphocytes and CD14 + monocytes/macrophages. Within 3 days of induction in the MSCs injected in articular arthropathy animals, a significant decrease in the numbers of circulating neutrophils in the blood and the concentration of activin A in the blood plasma was observed. From the results obtained, it was concluded that MSCs isolated from the ovaries can soften the clinical signs of arthritis and reduce the concentration of certain inflammatory mediators in the blood responsible for the destruction of joint tissue (Abdalmula et al., 2017).

In a model of medial condyle defect of the femoral bone and trochlear notch in goats, MSCs-AT therapy and stromal vascular fraction (SVF) were used. The cells were seeded on a type I/III collagen scaffold and transplanted into animals. This procedure led to 
stimulation and regeneration of cartilage compared to animals treated with scaffolds of cell-free collagen I/III. The researchers reported that there were no significant differences in the rate of regeneration between animals that received a collagen scaffold with MSCs-AT or SVF (Jurgens et al., 2013).

\section{Regenerative therapy of cartilage injury in dogs by} MSCs transplantation

In contrast to most studies in goats and sheep, dogs are not only used as model animals in preclinical research when developing MSCs therapies, but also as speciesspecific treatments which are being developed for their own healthcare needs (Kazemi et al., 2017).

Kazemi et al. (2017) conducted a series of experiments on 12 adult dogs in which osteochondral defects were created on the medial condyles of the femoral bone, into which MSCs-BM mixed with a thrombocyte mass were placed. In control animals, the defect was not filled in. According to the results of the experiment, the use of autologous cultures of MSCs-BM with thrombocyte masses stimulated cartilage regeneration in dogs. Thrombocyte masses are rich with fibrin and create a suitable environment for proliferation and differentiation of MSCs by releasing endogenous growth factors that lead to the creation of hyaline-like reparative tissue (Kazemi et al., 2017).

The treatment of nine dogs with natural OA on the background of joint dysplasia also showed interesting results. The animals had not received any treatment for 2 months prior to a single intra-articular injection of autologous MSCs-AT. Observations noted over the ensuing 6 months showed that lameness and severe pain disappeared for 3 months, but thereafter the symptoms of the disease began to reappear (Vilar et al., 2014). However, studies conducted by Shah et al. (2018) have shown a positive effect of intravenous or intra-articular administration of MSCs in dogs with natural degenerative arthritis. Of the 203 dogs treated, $88 \%$ had a significant reduction in clinical symptoms, while the remaining $12 \%$ had no reaction following cell administration. At the same time, it should be noted that the positive effects of the introduced MSCs-AT also correlated with the age of the animals. Significant improvements occurred in $90 \%$ of the younger dogs (up to 9 years old), which reduced to $60 \%$ of older dogs (Shah et al., 2018).

Intra-articular introduction of allogeneic MSCs in $0.5 \%$ hyaluronic acid to dogs suffering from elbow joint dysplasia and OA showed significant improvements in the condition of animals. Researchers noted the absence of lameness or reduction to just low-grade lameness for 1 year. Control arthroscopy of the first group of dogs with the absence of lameness showed that the cartilage was restored. Histological analyses of the cartilage biopsy also confirmed that the regenerated cartilage was of the hyaline type. These results demonstrate that allogeneic MSCs-AT transplantation is a new, non-invasive, and highly effective therapeutic tool in the treatment of canine dysplasia of the elbow joint (Kriston-Pal et al., 2017).

The influence of species-specific MSCs umbilical cords of dogs was studied in animals with naturally occurring OA in the elbow joint. The presence of changes in the dog's joint following treatment was confirmed by computer tomography. After collecting CB from dogs during planned cesarean section, MSCs were isolated from the umbilical cord and cultured in vitro. Each of the 28 dogs was given 7 million cells intra-articularly, and the 28 control dogs were given a saline solution. None of the dogs in either group developed worse lameness after treatment; therefore, none of the dogs had to be removed from the study. No adverse local side effects (increased pain, the appearance or increase of edema, and the appearance of secretions from the joints) were observed. The overall results of this study demonstrated an improvement of clinical signs over 6 months in dogs treated with intra-articular injections of the umbilical cord. This fact is very attractive because other treatments of OA, such as corticosteroids, lead to risks associated with cytotoxicity, anaphylactic reactions, or septic arthritis (Kim et al., 2019).

\section{Therapy of cartilage injury MSCs in horses}

Most studies of MSC treatment in horses, whether preclinical or clinical, indicate incomplete regeneration of damaged horse cartilage, but MSCs therapy did produce a decrease in the severity of clinical signs in these animals (Broeckx Sarah et al., 2019; Kovac et al., 2018). According to literature data, animal recovery was more effective when MSCs were introduced in the early stages of the disease but decreased when introduced at later stages (Wilke et al., 2007). Similar to autologous MSCs, a single intra-articular transplantation of allogeneic MSCs-BM also did not stir an immune response (Ardanaz et al., 2016), but repeated intra-articular transplantation led to adverse reactions following the introduction of allogeneic MSCs (Joswig et al., 2017).

MSCs-BM and AT have contributed toward the regeneration of damaged meniscus of horses, by restoring cartilage tissue (Gonzalez-Fernandez et al., 2016). In this model of horse femoral knee disease, MSCs seeded in an autogenously self-polymerizing fibrin carrier were used with better results in the early stages compared to a cell-free fibrin carrier. In equids, improvements in arthroscopic parameters of the damaged horse meniscus cartilage were observed 30 days after MSCs transplantation (Kovac et al., 2018). However, after a longer follow-up period ( 8 months), histological indicators in the group with MSCs were comparable to the control group (Wilke et al., 2007). Nevertheless, after 12 months, there was a slight improvement in clinical and histological indicators compared to the control group animals. In general, the use of MSCs-BM resulted in improved cartilage quality (McIIwraith et al., 2011). The ability of MSCs$\mathrm{BM}$ to stimulate the regeneration of cartilage tissue is 
considered to be better than that of the SVF of adipose tissue (SVF-AT). The use of MSCs-BM in comparison with SVF-AT leads to better clinical, biochemical, and histological results in the treatment of horse joints with OA on day 70 following treatment (Frisbie et al., 2009). In the search for effective treatments for degenerative joint disease, Broeckx Sarah et al. (2019) conducted a study involving 75 adult horses that had early signs of joint damage. Fifty animals were given intravenous allogeneic MSCs induced in the chondrogen direction with equine allogeneic plasma, and 25 horses were given a control drug having $0.9 \% \mathrm{NaCl}$. Long-term observation of animals after MSCs transplantation (1 year) showed that a significant number of horses subjected to cell therapy worked at the training level or returned to the previous level (Broeckx Sarah et al., 2019).

Due to the presence of a considerable number of sources of MSCs, Zayed et al. (2018) conducted comparative studies of MSCs-BM and MSCs from synovial fluid (MSCs-SF) of horses in vitro. The results showed that MSCs-SF had similar proliferative activities to MSCs-BM and expressed the membrane markers CD29, CD44, and CD90, but demonstrated different chondrogenesis characteristics. In addition to this, MHC II was positively expressed in all MSCs, while in MSCs-BM the expression was negative. When both autologous and allogeneic MSCs were administered in vivo, an increment in the level of total protein and in the total number of nucleated cells was observed in the horse's ankle joint. Therefore, further experiments to assess in vivo acute or chronic responses to allogeneic or autologous MSCs from various sources are necessary (Zayed et al., 2018).

\section{Conclusion}

The articular cartilage after damage tends to deteriorate every day due to its typical location and limited innate healing potential. With limited success of modern surgical methods, the inclusion of stem cells in regenerative drug therapy is intensively studied to ensure better rehabilitation of cartilage. Various stem cells derived from AT and BM MSCs are studied to assess their clinical use. This technology promises to develop a mechanically strong border between cartilage and cartilage, and includes mesenchymal condensation into cell bodies under the influence of growth factors. However, this method is yet to be tested in vivo (clinical conditions). The clinical use of MSCs has mainly been applied in dogs and horses, while in sheep and goats MSCs has mainly been studied in preclinical experimental models. MSCs chondrogenesis greatly varies in terms of cell sources, methods of cultivation, number of passages, number of implants required, and the inclusion of growth factors, and hence requires further research. In general, the best treatment is observed in the treatment of MSCs compared to the control. However, there are various problems with the treatment of MSCs, such as the lack of hyaline tissue regeneration, integration of the regenerated tissue matrix with the native cartilage or subchondral bone of the host, and its comparable effectiveness in all cases. Thus, the use of the unique regenerative properties of MSCs from autologous AT, BM, CB, CT, and WJ can be considered fully effective, clinically applicable, safe, and dependable for improving the clinical results and therapeutic doses of injected cells.

\section{Conflict of interest}

The authors declare that there is no conflict of interest.

\section{Acknowledgments}

The reported study was funded by the RFBR (project number 20-016-00022) and by the Russian Government Program of Competitive Growth of Kazan Federal University.

\section{References}

Abdalmula, A., Dooley, L.M., Kaufman, C., Washington, E.A., House, J.V., Blacklaws, B.A., Ghosh, P., Itescu, S., Bailey, S.R. and Kimpton, W.G. 2017. Immuno-selected STRO-3p mesenchymal precursor cells reduce inflammation and improve clinical outcomes in a large animal model of monoarthritis. Stem Cell Res. Ther. 8(1), 22.

Al Faqeh, H., Nor Hamdan, B.M.Y., Chen, H.C., Aminuddin, B.S. and Ruszymah, B.H.I. 2012. The potential of intra-articular injection of chondrogenic-induced bone marrow stem cells to retard the progression of osteoarthritis in a sheep model. Exp. Gerontol. 47(6), 458-464.

Ando, W., Heard, B.J., Chung, M., Nakamura, N., Frank, C.B. and Hart, D.A. 2012. Ovine synovial membrane-derived mesenchymal progenitor cells retain the phenotype of the original tissue that was exposed to in-vivo inflammation: evidence for a suppressed chondrogenic differentiation potential of the cells. Inflamm. Res. 61(6), 599-608.

Ardanaz, N.V., Vazquez, F.J., Romero, A., Remacha, A.R., Barrachina, L., Sanz, A., Ranera, B., Vitoria, A., Albareda, J., Prades, M., Zaragoza, P., MartínBurriel, I. and Rodellar, C. 2016. Inflammatory response to the administration of mesenchymal stem cells in an equine experimental model: effect of autologous, and single and repeat doses of pooled allogeneic cells in healthy joints. BMC Vet. Res. 12(1), 65.

Bornes, T.D., Adesida, A.B. and Jomha, N.M. 2018. Articular cartilage repair with mesenchymal stem cells after chondrogenic priming: a pilot study. Tissue Eng. Part A. 24(9-10). 761-774.

Broeckx Sarah, Y., Seys, B., Suls, M., Vandenberghe, A., Marien, T., Adriaensen, E., Declercq, J., Van Hecke, L., Braun, G., Hellmann, K. and Spaas, J.H. 2019. Equine allogeneic chondrogenic induced mesenchymal stem cells are an effective treatment 
for degenerative joint disease in horses. Stem Cells Dev. 28(6), 410-422.

Caminal, M., Fonseca, C., Peris, D., Moll, X., Rabanal, R.M., Barrachina, J., Codina, D., Garc, F., Cair, J.J., Godia, F., Pla, A. and Vives, J. 2014. Use of a chronic model of articular cartilage and meniscal injury for the assessment of long-term effects after autologous mesenchymal stromal cell treatment in sheep. New Biotechnol. 31(5), 492-498.

Duarte Campos, D.F., Drescher W., Rath, B., Tingart, M. and Fischer, H. 2012. Supporting biomaterials for articular cartilage repair. Cartilage. 3(3), 205221.

Feng, C., Luo, X., He, N., Xia, H., Lv, X., Zhang, X., Li, D., Wang, F., He, J., Zhang, L., Lin, X., Lin, L., Yin, H., He, J., Wang, J., Cao, W., Wang, R., Zhou, G. and Wang, W. 2018. Efficacy and persistence of allogeneic adipose-derived mesenchymal stem cells combined with hyaluronic acid in osteoarthritis after intra-articular injection in a sheep model. Tissue Eng. Part A. 24(3-4), 219-233.

Francis, S.L., Di Bella, C., Wallace, G.G. and Choong, P.F.M. 2018. Cartilage tissue engineering using stem cells and bioprinting technology-barriers to clinical translation. Front. surg. 5(70), 1-12.

Frisbie, D.D., Cross, M.W. and McIlwraith, C.W. 2006. A comparative study of articular cartilage thickness in the stifle of animal species used in human preclinical studies compared to articular cartilage thickness in the human. Vet. Comp. Orthop. Traumatol. 19(03), 142-146.

Frisbie, D.D., Kisiday, J.D., Kawcak, C.E., Werpy, N.M. and Mcllwraith, C.W. 2009. Evaluation of adipose-derived stromal vascular fraction or bone marrow-derived mesenchymal stem cells for treatment of osteoarthritis. J. Orthop. Res. 27(12), $1675-1680$.

Gao, Y., Zhu, Z., Zhao, Y., Hua, J., Ma, Y. and Guan, W. 2015. Multilineage potential research of bovine amniotic fluid mesenchymal stem cells. Int. J. Mol. Sci. 15(3), 698-710.

Gonzalez-Fernandez, M.L., Perez-Castrillo, S., Sanchez-Lazaro, J.A., Prieto-Fernandez, J.G., Lopez-Gonzalez, M.E., Lobato-Perez, S., Colaco, B.J., Olivera, E.R. and Villar-Suarez, V. 2016. Assessment of regeneration in meniscal lesions by use of mesenchymal stem cells derived from equine bone marrow and adipose tissue. Am J. Vet. Res. 77(7), 779-788.

Gugjoo, M.B., Amarpal, Sharma, G.T., Aithal, H.P. and Kinjavdekar, P. 2016. Cartilage tissue engineering: role of mesenchymal stem cells along with growth factors and scaffolds. Indian J. Med. Res. 144, 339347.

Jean, G.S. and Anderson, D.E. 2014. Decision analysis for fracture management in cattle. Vet. Clin. North Am. Food Anim. Pract. 30(1), 1-10.
Joswig, A.J., Mitchell, A., Cummings, K.J., Levine, G.J., Gregory, C.A., Smith, R. and Watts, A.E. 2017. Repeated intra-articular injection of allogeneic mesenchymal stem cells causes an adverse response compared to autologous cells in the equine model. Stem Cell Res. Ther. 8(1), 42.

Juneau, C., Paine, R., Chicas, E., Gardner, E., Bailey, L. and McDermott, J. 2016. Current concepts in treatment of patellofemoral osteochondritis dissecans. Int. J. Sports Phys Ther. 11(6), 903-925.

Jurgens, W., Kroeze, R.J., Zandieh-Doulabi, B., Van Dijk, A., Renders, G.A., Smit, T.H., Van Milligen, F.J., Ritt, M.J. and Helder, M.N. 2013. One-step surgical procedure for the treatment of osteochondral defects with adipose-derived stem cells in a caprine knee defect: a pilot study. Biores. Open Access. 2(4), 315-325.

Kazemi, D., Shams Asenjan, K., Dehdilani, N. and Parsa, H. 2017. Canine articular cartilage regeneration using mesenchymal stem cells seeded on platelet rich fibrin. Bone Joint Res. 6(2), 98-107.

Kim, S.E., Pozzi, A., Yeh, J., Lopez-Velazquez, M., Au Yong, J.A., Townsend, S., Dunlap, A.E., Christopher, S.A., Lewis, D.D., Johnson, M.D. and Petrucci, K. 2019. Intra-articular umbilical cord derived mesenchymal stem cell therapy for chronic elbow osteoarthritis in dogs: a double-blinded, placebo-controlled clinical trial. Front. Vet. Sci. 6, 474.

Kovac, M., Litvin, Y.A., Aliev, R.O., Zakirova, E.Y., Rutland, C.S., Kiyasov, A.P. and Rizvanov, A.A. 2018. Gene therapy using plasmid DNA encoding VEGF164 and FGF2 genes: a novel treatment of naturally occurring tendinitis and desmitis in horses. Front. Pharmacol. 9, 978.

Kriston-Pal, E., Czibula, A., Gyuris, Z., Balka, G., Seregi, A., Sükösd, F., Süth, M., Kiss-Tóth, E., Haracska, L., Uher, F. and Monostori, É. 2017. Characterization and therapeutic application of canine adipose mesenchymal stem cells to treat elbow osteoarthritis. Can. J. Vet. Res. 81, 73-78.

McIIwraith, C.W., Frisbie, D.D., Rodkey, W.G., Kisiday, J.D., Werpy, N.M., Kawcak, C.E. and Steadman, J.R. 2011. Evaluation of intraarticular mesenchymal stem cells to augment healing of microfractured chondral defects. Arthroscopy. 27, 1552-1561.

Mount, N.M., Ward, S.J., Kefalas, P. and Hyllner, J. 2015. Cell-based therapy technology classifications and translational challenges. Philos. Trans. R. Soc. Lond. B. Biol. Sci. 370(1680), 1-16.

Nifontov, K.R. 2009. Morphofunctional characteristic and the ways of the destructive joint changes correction in the sports horses: experimentalclinical investigation [in Russian]. PhD Dissertation of Veterinary Sciences. Moscow, Russia.

Pigareva, Yu.V. 2016. Clinical and morphological substantiation of efficiency of platelet-enriched 
autoplasm use at aseptic dog osteoarthritis [in Russian]. PhD dissertation of veterinary sciences. Saratov, Russia.

Ragni, E., Perucca Orfei, C., De Luca, P., Mondadori, C., Viganò, M., Colombini, A. and De Girolamo, L. 2020. Inflammatory priming enhancers mesenchymal stromal cell secretome potential as a clinical product for regenerative medicine approaches through secret factors and EV-miRNAs: the example of joint disease. Stem Cell Res. Ther. 11(1), 165.

Redman, S.N., Oldfield, S.F. and Archer, C.W. 2005. Current strategies for articular cartilage repair. Eur. Cell Mater. 9, 23-32.

Shah, K., Drury, T., Roic, I., Hansen. P., Malin, M., Boyd, R., Sumer, H. and Ferguson, R. 2018. Outcome of allogeneic adult stem cell therapy in dogs suffering from osteoarthritis and other joint defects. Hindawi. Stem Cells Int. 272, 1-7.

Tiwary, R., Amarpal, Aithal, H.P., Kinjavdekar, P., Pawde, A.M. and Singh, R. 2014. Effect of IGF1 and uncultured autologous bone-marrow-derived mononuclear cells on repair of osteochondral defect in rabbits. Cartilage 5, 43-54.

Toh, W.S., Brittberg, M., Farr, J., Foldager, C.B., Gomoll, A.H., Hui, J.H., Richardson, J.B., Roberts, S. and Spector, M. 2016. Cellular senescence in aging and osteoarthritis. Acta Orthop. 87(Sup363), 6-14.

Vega, A., Martin-Ferrero, M.A., Del Canto, F., Alberca, M., Garcia, V., Munar, A., Orozco, L., Soler, R., Fuertes, J.J., Huguet, M., Sánchez, A. and GarcíaSancho, J. 2015. Treatment of knee osteoarthritis with allogeneicbone marrow mesenchymal stem cells: arandomized controlled trial. Transplantation. 99(8), 1681-1690.
Vilar, J.M., Batista, M., Morales, M., Santana, A., Cuervo, B., Rubio, M., Cugat, R., Sopena, J. and Carrillo, J.M. 2014. Assessment of the effect of intraarticular injection of autologous adiposederived mesenchymal stem cells in osteoarthritic dogs using a double blinded force platform analysis. BMC Vet. Res. 10(1), 143.

Wilke, M.M., Nydam, D.V. and Nixon, A.J. 2007. Enhanced early chondrogenesis in articular defects following arthroscopic mesenchymal stem cell implantation in an equine model. J. Orthop. Res. 25(7), 913-925.

Zakirova, E.Yu., Valeeva, A.N., Aimaletdinov, A.M., Nefedovskaya, L.V., Akhmetshin, R.F., Rutland, C.S. and Rizvanov, A.A. 2019. Potential therapeutic application of mesenchymal stem cells in ophthalmology. Exp. Eye Res. 189, 107863.

Zakirova, E.Yu., Valeeva, A.N., Masgutov, R.F., Naumenko, E.A. and Rizvanov, A.A. 2017. Application of allogenic adipose-derived multipotent mesenchymal stromal cells from cat for tibial bone pseudoarthrosis therapy (Case Report). BioNanoScience. 7, 207-211.

Zayed, M.N., Adair, S., Ursini, T., Schumacher, J., Misk, N. and Dhar, M.S. 2018. Concepts and challenges in the use of mesenchymal stem cells as a treatment for cartilage damage in the horse. Res. Vet. Sci. 118, 317-323.

Zayed, M.N., Schumacher, J., Misk, N. and Dhar, M.S. 2016. Effects of pro-inflammatory cytokines on chondrogenesis of equine mesenchymal stromal cells derived from bone marrow or synovial fluid. Vet. J. 217, 26-32. 